\title{
Advising patients about breast implant associated anaplastic large cell lymphoma
}

\author{
Christopher S. Parham¹, Summer E. Hanson ${ }^{2}$, Charles E. Butler ${ }^{2}$, M. Bradley Calobrace ${ }^{3}$, \\ Raylene Hollrah ${ }^{4}$, Terri Macgregor ${ }^{4}$, Mark W. Clemens ${ }^{2}$
}

${ }^{1}$ Department of Surgery, Division of Plastic Surgery, Pennsylvania State University, College of Medicine, Hershey, PA, USA; ${ }^{2}$ Department of Plastic Surgery, MD Anderson Cancer Center, University of Texas, Houston, TX, USA; ${ }^{3}$ CaloAesthetics Plastic Surgery Center; Clinical Faculty Member, Divisions of Plastic Surgery, University of Louisville and University of Kentucky, Louisville and Lexington, KY, USA; ${ }^{4}$ Breast Implant Lymphoma Advocates (BILA), Hermann, MO, USA

Contributions: (I) Conception and design: All authors; (II) Administrative support: MW Clemens, CE Butler; (III) Provision of study materials or patients: MW Clemens; (IV) Collection and assembly of data: CS Parham, MW Clemens; (V) Data analysis and interpretation: CS Parham, MW Clemens; (VI) Manuscript writing: All authors; (VII) Final approval of manuscript: All authors.

Correspondence to: Mark W. Clemens, MD. Department of Plastic Surgery, MD Anderson Cancer Center, University of Texas, 1400 Pressler St. Unit 1488, Houston, TX, USA. Email: mwclemens@mdanderson.edu.

\begin{abstract}
The United States Food and Drug Administration (FDA) recently recognized the necessity for improved patient education and public awareness of breast implant-associated anaplastic large cell lymphoma (BIA-ALCL). BIA-ALCL is an uncommon T-cell lymphoma that can arise years after placement of a textured breast implant or tissue expander. Patients and providers would greatly benefit from a broader awareness of BIA-ALCL and how it presents with both common and rare symptoms. Disease awareness can be improved with specific steps moving forward. This includes improved counseling about annual recommendations from the FDA, standard of care guidelines, current estimates of disease prevalence, and manufacturer-specific risk for developing the malignancy. Informed consent for implant-based breast surgery should include a discussion of BIA-ALCL, symptoms, inherent risks and alternatives. Consideration should be given for reasonable efforts to retroactively contact patients with textured implants to inform them of recent regulatory actions as well as the risk of developing the disease and the signs and symptoms to watch for. Where risk can be reduced, all steps should be taken to ensure patient safety. In keeping with our commitment to patient safety, the strategies moving forward for implant-based breast surgery should be marked by patient awareness, physician vigilance, and defined by evidence-based diagnosis and treatment.
\end{abstract}

Keywords: Breast implants; device safety; breast implant-associated anaplastic large cell lymphoma (BIA-ALCL); breast implant associated; informed consent

Submitted Feb 05, 2020. Accepted for publication Feb 28, 2020.

doi: 10.21037 /gs.2020.03.06

View this article at: http://dx.doi.org/10.21037/gs.2020.03.06

\section{Introduction}

Recent studies on the long term outcomes of breast implants prompted the Food and Drug Administration (FDA) to convene a 2019 advisory committee on the safety of breast implants (1). In an effort to protect women's health and enhance safety information available to patients, the FDA called for a number of actionable steps to address breast implant risk and in particular breast implantassociated anaplastic large cell lymphoma (BIA-ALCL). BIA-ALCL is an iatrogenic malignancy, associated with breast surgery involving textured surface breast implants and tissue expanders. BIA-ALCL first came to the limited attention of the medical community in 1997 from an initial case report which eventually triggered significant interest and subsequent research (2). In 2011, the FDA reported 34 
Table 1 Common and subtle symptoms of BIA-ALCL are listed

\begin{tabular}{lll}
\hline Common symptoms & Uncommon symptoms $(<5 \%)$ & Patient observed symptoms (self described) \\
\hline Delayed seroma/effusion (> one year from implantation) & Fever, night sweats (B symptoms) & Rapid unilateral breast enlargement \\
Palpable mass & Pruritic skin rashes/lesions & Change in breast shape \\
Capsular contracture & Pain & Deep/internal itching sensation \\
Lymphadenopathy & & Fatigue \\
\hline
\end{tabular}

Feedback from patients demonstrates patients or surgeons may not be aware of subtle symptoms. Any unexplained breast irregularity should lead a patient to seek out evaluation by her health care provider. BIA-ALCL, breast implant-associated anaplastic large cell lymphoma.

unique cases worldwide bringing BIA-ALCL to the initial attention of most physicians and the general public $(3,4)$. BIA-ALCL has since been recognized as a distinct cancer by international organizations after data collection from multiple ongoing registries (5). In the 9 years since their initial warning, the FDA has continued to provide periodic updates, and at the time of writing this article, recognized 573 distinct and pathologically confirmed cases worldwide, with 33 associated deaths (6). Recent critical scrutiny led to a 2019 US FDA Class I device recall and a subsequent voluntary worldwide recall from the highest volume textured implant manufacturer, Allergan Corporation (Dublin, Ireland).

Based upon recent publications, the academic community now recognizes a distinct opportunity to do more to provide the most accurate and transparent information to women who are considering breast implants, and to confront the ethical implications and resulting obligations of inserting a device which carries a known risk of BIA-ALCL. In light of the rapidly changing and growing body of evidence, patients need ongoing access to the most current evolving information so that they may understand an increasing risk assessment and surgical options in order to make informed decisions. Patients who choose to have breast implants have a right to be active participants in their care, which can only be achieved with appropriate and comprehensive counseling. The current landscape for patient counseling is an opportunity to re-establish trust for patients with existing textured implants who are seeking information about the potential need for a prophylactic explantation for their exposure to a possible carcinogen. Additionally, patients rely on plastic surgeons to take the lead in providing much-needed education and guidance to all of their multidisciplinary colleagues involved in patient care, specifically radiology, medical oncology, breast surgery, and pathology. While we cannot undue the risk posed to the patient population exposed to textured implants, we as plastic surgeons can move forward to educate in providing the best and most accurate information to the patient community demanding transparency on medical devices. Patient advocates recommend an honest and transparent approach when counselling patients seeking guidance on implant concerns. The purpose of this manuscript is to outline the critical features that should be a part of a comprehensive discussion with patients concerning issues related to BIA-ALCL and implant-based breast surgery.

\section{Clinical features}

\section{Presentation}

Breast implant patients and their caregivers in the medical community need to know common and rare presenting symptoms of BIA-ALCL, and what further action should be taken when they occur. BIA-ALCL is a preventable non-Hodgkin's T-Cell lymphoma which presents as an effusion or mass, most often in a delayed fashion (occurring greater than one year after implantation) (4,7-9). Patients usually experience rapid onset and spontaneous unilateral breast enlargement, found to be a fluid collection or infiltrating mass at an average of 8 to 10 years after implant placement, with the shortest interval to date being 2.2 years from initial implantation $(10,11)$. Other reported symptoms may include a change in breast shape, breast firmness, pain, pruritic skin rashes or lesions, fever, night sweats, fatigue, capsular contracture, or lymphadenopathy $(12,13)$. At presentation, patients have less commonly described a "deep-seeded, internal itching" (see Table 1). Any unexplained breast irregularity should lead a patient to seek out evaluation by their health care provider. While delayed seromas are the most common presentation, there 
is a frequent misconception that absence of fluid excludes a diagnosis. The disease is most commonly confined to the periprosthetic space, but there are cases with more advanced features such as chest wall invasion, bilateral disease, lymph node involvement, and organ metastasis, often refractory to surgical treatment alone (7).

\section{Textured versus smooth implant surfaces}

BIA-ALCL is a disease associated with an exposure to textured surface implants. The exact etiology remains unclear, and is most likely multifactorial (13-18). To date, there have been no identified cases reported in any series, case study, or registry with a history of only smooth devices $(6,19)$. All other forms of ALCL (systemic and primary cutaneous) occur with an approximate frequency of one in four million within the general population and therefore it is important to distinguish BIA-ALCL from primary lymphoma of the breast or systemic ALCL coincidentally occurring in a patient with breast implants. It is important to note that the FDA does report 26 cases of BIA-ALCL arising in patients with smooth implants. But of those 26 cases, 7 had previous textured devices, while the remaining 19 patients had an unknown history of prior implants. One death has been reported in association with smooth implants, but the patient had a history of prior textured devices (6). Therefore, a clinical history of textured implant exposure, even with device removal, appears to be sufficient to lead to future disease in some patients. Previous exposure to textured devices should be included in patient discussions about potential prophylactic removal or implant exchange. Some surgeons have found logic in removing the capsule and textured implant especially if the capsule can be completely removed such as in a subglandular implant, however to date there is no current evidence that removal/ exchange of textured implants or total capsulectomy eradicates future risk of BIA-ALCL. While there is a risk of morbidity with total capsulectomy, in cases in which BIAALCL is present and clinically unknown, inadvertent partial resection may lead to progression of the disease.

Patients need to be made aware of the available surface types used in implants and the specific type that was utilized for their surgery. No prospective randomized controlled trial exists of modern smooth versus textured implants in the last twenty years demonstrating the superiority of one implant over the other in aesthetic outcomes or risk reduction and several studies have demonstrated that both plastic surgeons and patients have difficulty appreciating the aesthetic differences between the two types (20-23).

Implant surfaces are not binary, such as smooth versus textured, as many different manufacturer-specific texturing exists. Risks from textured devices, discussed below, are not the same across all texture types but rather exist on a spectrum demonstrating variable implant specific risk (24-26). The specific risks of many manufacturers worldwide remain unknown. Patients with breast implants have the right to know about their operative history and the type or types of implants that have been utilized, and new prospective patients considering breast implant surgery can only electively accept the associated risks if adequately informed (26).

\section{Diagnosis and treatment}

Patients with a history of breast implants, irrespective of surface type, presenting with a delayed onset seroma, sudden unilateral breast enlargement, or mass warrant careful further evaluation. Trauma to breast and mastectomy for breast cancer have coincidentally led to BIA-ALCL diagnosis, therefore a history of breast trauma with these findings does not absolutely rule-out the diagnosis of BIA-ALCL. Symptoms of infection can also mimic the symptoms of BIA-ALCL, and thus should be thoroughly evaluated before dismissal of malignancy. Of course, a history of textured device should increase one's suspicion during the evaluation. The diagnosis is made with either ultrasound or MRI imaging and pathological analysis of fluid and/or tissue samples $(12,13,27,28)$.

Periprosthetic fluid aspirate sent only for routine pathology evaluation may be incorrectly labeled benign if CD30 immunohistochemistry is not specifically ordered. There have been many incidences where the diagnostic studies were not properly performed or interpreted leading to a missed or late diagnosis. Patient Advocates recommend patients review their pathology report and (CD30 immunohistochemistry) to ensure accurate evaluation was performed for both fluid and capsule tissue after biopsies and surgery (29). Communication is essential between surgeons and pathologists, to make sure the type of evaluation is clear, particularly when all parties may be unfamiliar with the disease. For community-based practices, pathology reports that are unclear or inconclusive may be referred to a tertiary cancer center for further evaluation. As a cautionary note, diagnosed patients have been misdiagnosed with a ruptured implant, yet upon explantation the implant is found intact but with a large 
surrounding malignant effusion.

The management of all confirmed BIA-ALCL patients initially involves imaging for metastasis with a PETCT scan and oncology consultation, followed by surgical excision. This involves an oncologic en bloc resection, with implant removal, total capsulectomy, and tumor excision with negative margins (30). If there is residual disease or there is evidence of invasion beyond the capsule, adjuvant therapies may be required such as systemic chemotherapy, targeted immune therapy, chest wall radiation, and/or stem cell transplant (31).

Patients similarly should know that with early detection and adequate treatment, outcomes are encouraging. Multiple studies indicate overall survival rates greater than $90 \%$ if detected in early stages $(10,25,32)$. Conversely, a delay in diagnosis is associated with advanced disease, regional and distant metastasis, and disease-related death (7). Early-stage BIA-ALCL patients have a higher rate of definitive surgery, while advanced-stage patients overall have a lower incidence of definitive surgery, more adjuvant therapy including chemotherapeutics, and a higher rate of autologous stem cell transplants (7). Current data suggests that if diagnosed early, BIA-ALCL is easier to manage and is associated with better outcomes. Treatment in those that are detected late is more complicated and overall portends worse prognosis. Thus, ongoing surveillance of all patients with breast implants is imperative for early detection, leading to better overall prognosis and survival.

\section{Evaluating risk}

\section{Current risk estimates}

Patients need to have a firm understanding of the overall risk of developing BIA-ALCL. This includes patients whom already have breast implants so they can thoroughly understand their risk associated with their specific type of breast implant, which may include not only their current implant but also all previous implants. In many of these patients, these risks were not known or were not discussed with the patient at the time of implantation. New patients considering breast implant-based surgery should likewise be thoroughly counseled to insure both risks and benefits are understood. Implant-based breast surgery is elective, but the risk incurred is only elective if the patient is adequately informed. A distinction should be made when addressing disease incidence versus prevalence, the latter referring to the number of cases in a population in a given time period (33).
The prevalence of BIA-ALCL has been difficult to assess due to inadequacy in reporting, incomplete patient records, and difficulty estimating the total number of patients with breast implants, but it is exponentially higher than initial estimates nine years ago. In 2011, the FDA first reported 34 unique cases $(3,4)$. As of November 2019, there are 573 distinct worldwide cases reported by the FDA. This represents a seventeen-fold increase in the number of reported cases in an eight-year period owing to the emerging nature of this disease. Much of this increase in cases is likely attributable to improved disease awareness and reporting. It is important to note that under-reporting still exists within many countries (34), coupled with poor access to modern healthcare and faulty patient records which have likely led to under-estimation of BIA-ALCL prevalence globally.

One recent study sought to determine the lifetime prevalence of BIA-ALCL in the U.S. The authors identified 100 patients diagnosed with BIA-ALCL amongst an estimated 3 million patients with textured breast implants over a nearly two-decade timeframe. They concluded that the lifetime prevalence was 1:30,000 for the U.S. population in 2017 (35). Importantly, 1:30,000 represented an average risk across at least two separate major manufacturers, Allergan and Mentor (Irvine, CA, USA), and that a 6:1 risk ratio was reported between these manufacturers within that study (Allergan implants with the greater risk). Recent findings collected from the BIA-ALCL Patient Registry and Outcomes for Breast Implants and Anaplastic Large Cell Lymphoma Etiology and Epidemiology (PROFILE) registry shed more light on current prevalence. The PROFILE registry is a collaboration between the American Society of Plastic Surgeons (ASPS), the Plastic Surgery Foundation (PSF), and the US FDA for the prospective collection of United States data to better characterize BIAALCL. In a report of their findings from 2012 to 2018, the registry identified 186 distinct cases of BIA-ALCL, only $48 \%$ of which had complete records (36). The PROFILE registry now recognizes 307 confirmed or suspected cases in the U.S, bringing the total worldwide cases to 876 as of January $14^{\text {th }}, 2020$ (37).

Risk estimation is difficult to determine, as it relies upon many factors, as well as epidemiological data. Patients need to understand that with current data, a range of estimates is the best that can be offered and this will continue to change with ongoing data collection. The largest series ever reported on textured implants, the Continued Access/ 
Continued Access Reconstruction/Revision Expansion (CA/CARE) clinical trial, which was a 10-year, multicenter study of Allergan Biocell textured implants initially identified 4 cases of BIA-ALCL out of 17,656 patients. Since publication, the lead author further identified a total of eight patients bringing the risk estimate in their large prospective multicenter cohort to 1:2,207 (38,39). A recent study conducted at Memorial Sloan-Kettering Cancer Center reported a single-surgeon experience of 6023 Allergan Biocell breast reconstructions and having a risk of 1:355 (40). Recent data from Australia and New Zealand provides further information as it relates to risk assessment. Magnusson et al. reported manufacturer related risks based upon 81 BIA-ALCL patients and yearly national implant sales data amongst three implant manufacturers with the largest market share, Allergan, Mentor, and Silimed (Rio de Janeiro, Brazil). They reported manufacturer-specific risks based upon this data: Silimed polyurethane, 1:2,832 (95\% CI, 1,582 to 5,673); Allergan Biocell, 1:3,345 (95 percent CI, 2,475 to 4,642); Mentor Siltex, 1:86,029 (95 percent CI, 15,440 to $1,301,759)$ (24). Relative to the U.S. market, their findings indicate a 25.7 to 1 increased risk between the use of Allergan Biocell to Mentor Siltex implants for the development of BIA-ALCL. Confirmed cases have similarly been reported from Silimed, Mentor, Sientra (Santa Barbara, CA, USA), Poly Implant Prothèse (formerly La Seyne-sur-Mer, France), Nagor (owned by GC Aesthetics, Dublin, Ireland), Polytech (Dieburg, Germany), and Eurosilicone (owned by GC Aesthetics, Dublin, Ireland). While it is difficult to determine exact risks because of reporting inefficiencies, geographic bias, sales data estimations, true implant prevalence, and misdiagnosis, the most recent current estimates of lifetime risk estimates range between 1:2,207-1:86,029 $(7,24,25,39)$.

In 2019, the US FDA reported that of the worldwide cases, the majority of BIA-ALCL cases (91\%) were associated with textured Allergan Biocell products when the manufacturer was known $(6,19)$. Of the 107 confirmed cases in the 2019 PROFILE registry publication, Mentor accounted for $12(11 \%)$ of cases and Sientra for none, while Allergan products accounted for two thirds of the registry with 72 cases (67\%). This is also a 6 to 1 ratio of Allergan cases to Mentor cases (37). Of the 573 FDA recognized cases, Mentor and Sientra accounted for $7 \%$ and $1 \%$, respectively and a 12.6 to 1 ratio between Allergan Biocell and Mentor Siltex (41). The rest of the cases were either unknown or mixed implant cases.

\section{The biocell recall}

In response to this data, the FDA requested that Allergan remove Biocell surface implants and tissue expander products from the US market due to the disproportionate risk of BIA-ALCL (42). Allergan responded with a voluntary worldwide recall of Biocell textured devices in July of 2019. Allergan sent a letter to US breast implant patients for which they had active contact information which explained the voluntary recall and offered a warranty program which included providing implants at no charge for the replacement to smooth devices (43). Several ongoing lawsuits have been filed for coverage of costs incurred from implant removal, citing "negligence" and "failure to warn" as claims for compensation $(44,45)$. While litigation is ongoing, patients should be made aware that most but not all insurance will cover treatment for BIA-ALCL, and some denials of treatment have been reported in cosmetic augmentation patients. Importantly, women make decisions on what type of insurance coverage to keep or change on an annual basis and this information provided to breast implant patients may allow proactive steps to obtain appropriate insurance coverage.

\section{FDA position on breast implant safety}

In addition to epidemiological data, patients also need to be aware of the FDA's stance on breast implant safety. The FDA is currently in the drafting stages of new guidance recommendations for breast implants package labeling and the informed consent process (46). These recommendations are designed to improve patient communication so that patients are better informed about the inherent risks implicit with implants. The pending recommendations act as a supplement to the FDA's "Saline, Silicone Gel, and Alternative Breast Implants" guidance, issued in November of 2006 (47). The new draft specifically provides recommendations for a boxed warning, a standardized patient decision checklist, and information on BIA-ALCL, which they now list as a risk of implant-based breast surgery (Figure 1). Currently, the FDA does not recommend prophylactic removal of textured devices for asymptomatic patients based on the relatively low risk of developing BIAALCL. For patients who are having high risk textured implants explanted, their exposure to BIA-ALCL risk is not completely eradicated and this should be clearly explained to the patient as part of the informed consent if contemplating explantation surgery. The FDA has restricted any further 
Appendix A: Boxed Warning Example

WARNING:

- Breast implants are not considered lifetime devices. The longer people have them, the greater the chances are that they will develop complications, some of which will require more surgery.

- Breast implants have been associated with the development of a cancer of the immune system called breast implant-associated anaplastic large cell lymphoma (BIA-ALCL). This cancer occurs more commonly in patients with textured breast implants than smooth implants, although rates are not well defined. Some patients have died from BIA-ALCL.

- Patients receiving breast implants have reported a variety of systemic symptoms such as joint paint, muscle aches, confusion, chronic fatigue, autoimmune diseases and others. Individual patient risk for developing these symptoms has not been well established. Some patients report complete resolution of symptoms when the implants are removed without replacement.

Proposed FDA boxed warning (47).

Figure 1 Proposed FDA breast implant boxed warning (47).

placement of Biocell textured and unused inventory should be returned to the manufacturer. They confirm that other breast implants currently approved within the US market remain acceptably safe and efficacious $(42,46)$.

\section{Implant choice}

Selection of an implant without texturing modifies one's risk of BIA-ALCL. In a recent online survey sent to American Society of Plastic Surgeons members, approximately $47.7 \%$ of respondents reported switching to exclusive smooth implant use due to awareness of BIA-ALCL (48). Less than half of the respondents deemed BIA-ALCL as a significant enough risk to change their practice. This is likely because of multiple reasons. There are various manufacturers in which the prevalence of disease is very low in comparison to Biocell textured Allergan, such as textured products manufactured by Sientra and Mentor (41). Patients currently may still accept a level of risk at the discretion of their surgeon. Patients should be aware that silicone gel implants are still in post-market evaluation. Patients should be aware that there has never been a BIA-ALCL case reported with smooth-only products and that all current manufacturers offer smooth breast implants.

It is currently unknown how global trends of implant selection have shifted in response to BIA-ALCL, but the logical follow-up to this data is: what amount of risk do we deem is acceptable? While it is impossible to eliminate all risks from surgery, it is certainly possible to control the risk associated with the use of certain textured devices. It bears repeating that there are currently no documented cases of BIA-ALCL in smooth-only implant patients with complete records that have ever been reported $(6,19)$. This does not mean that a case of ALCL could not occur in a patient with smooth-only devices, but one has not been reported to date. Regardless, current data and reports indicate a strong correlation with textured implants. The choice to use textured implants or smooth implants is solely determined by the surgeon and the patient. Risk of BIA-ALCL may be lowered by selecting a textured implant with a lower risk factor for BIA-ALCL, and mitigated by use of smooth devices.

\section{Informed consent}

Physicians, manufacturers, medical societies, and governmental health agencies have a responsibility to inform patients of the risk of BIA-ALCL. Implant insertion, whether for reconstruction or aesthetics purposes, is a purely elective surgery, which carries a measurable risk for BIA-ALCL 
development. Surgeons are obligated to disclose this risk to patients during the informed consent process in part because risk disclosure is a form of respect for patient autonomy (49). Informed consent, by its very nature, should be designed to protect patients from incomplete or inaccurate information, allowing them to fully weigh the risks, benefits, complications, and alternatives for their treatment.

\section{BIA-ALCL consent inclusion}

Despite current recommendations from our national plastic surgery societies and the FDA, it is unknown how often practitioners actually address BIA-ALCL in the consent process. In 2015, approximately 500 society meeting attendees were asked if BIA-ALCL was included as a risk during the consent process and between two thirds and three fourths of surgeons did not discuss BIA-ALCL while consenting for implant-based surgery (49).

Similarly, in 2016 Pittman et al. reported their findings from a formal electronic 19-question survey that was sent to over 10,000 U.S. and international board-certified plastic surgeons, gathering responses from 1,383 physicians (US, 715; International, 668) for a response rate of $13.5 \%$ (34). They found that while the majority of respondents have not had cases of BIA-ALCL in their practice, $9.5 \%$ have treated at least one case of BIA-ALCL and close to $20 \%$ reported having colleagues with BIA-ALCL in their practice. Interestingly, they found that only $26.9 \%$ of respondents counsel their patients about BIA-ALCL every time, while $24.3 \%$ never counsel patients about the disease. Overall, 36.4\% include BIA-ALCL in their consent. Their findings also highlight important international differences in consenting for implants. Australian, French, and German physicians were five times more likely to include BIAALCL in the consent as compared to their US counterparts, whereas Brazil, Mexico, and South Africa were more likely to not include the risk of malignancy in the consent (34).

\section{Elements of informed consent}

Effective informed consent should incorporate several critical elements. The patient's capacity and ability to make decisions after disclosure of risks should be assessed during an open and unbiased discussion of the indications, benefits, complications, and alternatives to surgery (50). This should be balanced with sensitivity to the practical limitations and unknown variables inherent in medical decision-making. As it pertains to BIA-ALCL, discussion of surgery and consent should address three primary objectives. The first objective should aim to educate the patient about the existence of this uncommon disease. BIA-ALCL is a type of nonHodgkin's lymphoma which arises in the periprosthetic tissue surrounding implants. The second objective is to provide information on clinical presentation and to assess patient understanding and comprehension. Most patients with BIA-ALCL present in a delayed fashion with a fluid effusion or mass, on average 8 to 10 years after surgery $(7,10,12,13,18)$. The third objective is to provide a road map of diagnosis and treatment for patients should they become symptomatic. This involves examination by a physician knowledgeable about BIA-ALCL. Fluid collections should be aspirated and sent for CD-30 immunohistochemistry, flow cytometry, and histology. Treatment depends upon extent of disease, but if detected early, surgical extirpation carries a good prognosis $(7,12,18,49,51)$.

\section{Manufacturer package inserts}

It is essential for patient autonomy to have access to information from regulatory bodies as well as manufacturers. Before the 2011 FDA report warning of the association between breast implants and BIA-ALCL, manufacturers did not include package inserts with language addressing specifically BIA-ALCL. The inserts mentioned the possible risk of connective tissue disease, autoimmune syndromes, and risks of cancers including breast, brain, lung, cervical/ vulvar, and other cancers including leukemia (52). Given the current body of evidence and the FDA's stance on BIA-ALCL and implant safety, manufacturers have been compelled to address the condition in package inserts, present within US package inserts since 2012 and internationally since approximately 2015. Currently, package inserts for FDA-approved devices all address the risk of BIA-ALCL and its association with breast implants (see Table 2) (53).

Allergan now also provides an "Acknowledgement of Informed Decision" page to their insert, addressing that use of their product is elective and that patients have been given adequate information in order to make an informed decision, a change from their 2009 insert (53). While discussion about BIA-ALCL risk based upon the best current literature is vital to informed consent, transparency from device manufacturers and the U.S. FDA about the condition further improves patient decision-making. 
Table 2 BIA-ALCL warning in breast implant package insert*

If you have breast implants you have a very small, but increased risk of developing breast implant associated anaplastic large cell lymphoma, or BIA-ALCL. BIA-ALCL is not breast cancer-it is a rare type of non-Hodgkin's lymphoma (cancer of the immune system). In most cases, BIA-ALCL is found in the scar tissue and fluid near the implant, but in some cases, it can spread throughout the body. In the cases that have been spread beyond the scar tissue and fluid near the implant, rare cases of death have been reported

Most patients were diagnosed with BIA-ALCL when they sought medical treatment for implant-related symptoms such as swelling, pain, lumps, or asymmetry that developed after their initial surgical sites were fully healed. In the cases known to FDA to date, BIA-ALCL was diagnosed years after the breast implant was placed. The earliest report was one year after implant placement and the latest was 23 years after the implant surgery. About half the cases occurred within the first 7 years after implant. BIA-ALCL was most often diagnosed in women who had textured implants. The textured implant may have been placed at the most recent surgery or at any other prior breast implant operation

If you develop swelling or pain around your breast implants, be sure to talk to your health care provider. Your health care provider should consider the possibility of BIA-ALCL if, after you have recovered from your breast implant operation, you later notice changes in the way your breast looks or feels-including swelling or pain around the implant. If your health care provider suspects BIA-ALCL, they will refer you to an appropriate specialist for evaluation which may involve obtaining fluid and some tissue samples from around your breast implant. If a diagnosis of BIA-ALCL is confirmed, the doctor will develop an individualized treatment plan for you. Because of the small number of cases worldwide and the variety of available treatment options, there is no single defined treatment. However, if you are diagnosed with BIA-ALCL, the National Comprehensive Cancer Network (NCCN) recommends removing the implant and the surrounding tissue

If you have breast implants you should monitor them and follow your routine medical care. You do not need to take any additional steps. It is not necessary to remove your breast implants if you have no symptoms without a diagnosis of BIA-ALCL

If you are diagnosed with BIA-ALCL, you can help the FDA understand the disease and effectiveness of treatment. You or your doctor should report all confirmed cases of BIA-ALCL to the FDA (https://www.fda.gov/Safety/MedWatch/). In some cases, the FDA may contact you for additional information. The FDA will keep the identities of the reporter and the patient confidential

In addition, if you are diagnosed with BIA-ALCL, talk to your doctor about reporting it to the PROFILE Registry (https://www.thepsf.org/ research/clinical-impact/ profile.htm). Every case of BIA-ALCL should be reported to the PROFILE Registry because this helps provide a better understanding of the disease

If you are considering breast implant surgery, you should discuss the risks and benefits with your health care provider. You may also visit the FDA's Breast Implants website for additional information https://www.fda.gov/MedicalDevices/ProductsandMedicalProcedures/ ImplantsandProsthetics/BreastImplants/ucm064106.htm

For additional information on FDA's analysis and review of BIA-ALCL, please visit: https://www.fda.gov/MedicalDevices/ ProductsandMedicalProcedures/ImplantsandProsthetics/Breastlmplants/ucm239995.htm

*, example is from Allergan Corp (Dublin, Ireland) (53). BIA-ALCL, breast implant-associated anaplastic large cell lymphoma.

\section{Contacting patients}

The imperative to inform patients may extend beyond the consent process for individual patients. However, the logistical complexities of implementing this led to poor adoption. Indeed, some institutions have succeeded in taking action to alert patients retroactively in order to make them aware of BIA-ALCL risk. Roberts et al. took on the difficult task of alerting all patients with breast implants placed at Penn State Hershey Medical Center from 1979 to 2017, informing 1,340 patients of the risk of BIA-ALCL. Patients were grouped by implant surface type, either smooth or textured, and letters specific to each group were drafted and sent to the patients. Both letter types mentioned the risk of BIA-ALCL and provided resources for further information about the condition. Additionally, multiple departments were in cooperation and a call line was set up for patient use. Consequently, 100 patients asked to be evaluated and 9 patients asked for prophylactic implant removal based upon BIA-ALCL risk (54).

In 2018 Memorial Sloan Kettering Cancer Center also chose to alert all breast implant patients by letter of the risk of BIA-ALCL in response to treating a series of institutional cases. Similarly, M. D. Anderson Cancer Center has also taken steps to inform patients with patient information booklets and written scripts for staff to use in order to answer questions about BIA-ALCL (39). A sample letter and a list of frequently asked questions was sent to all 

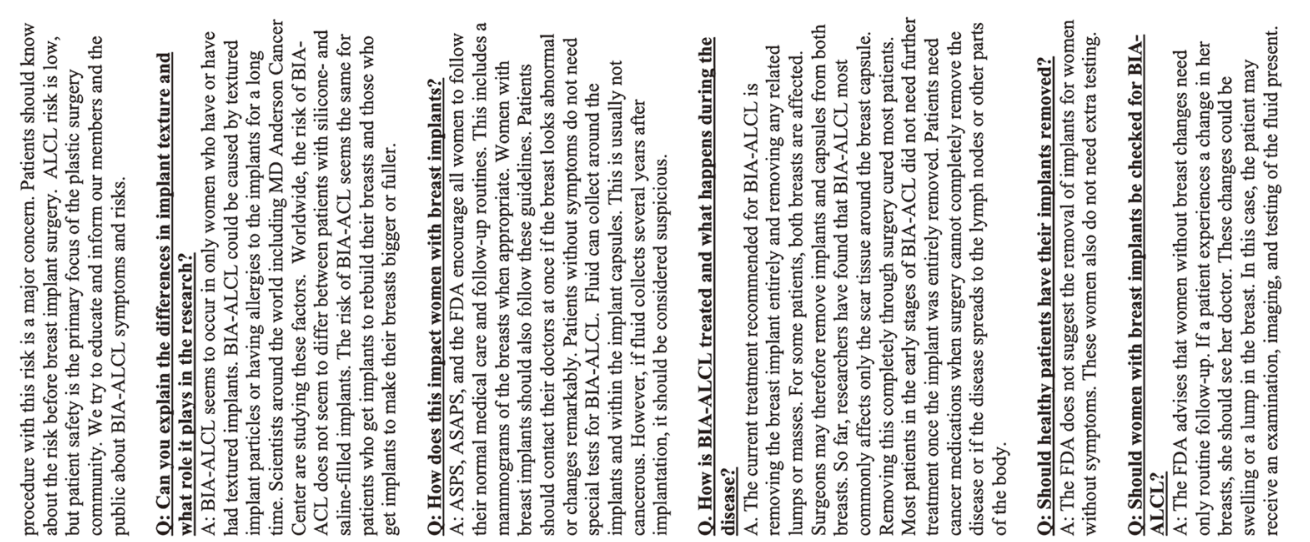

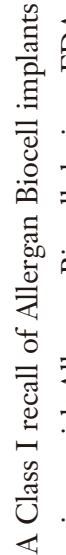
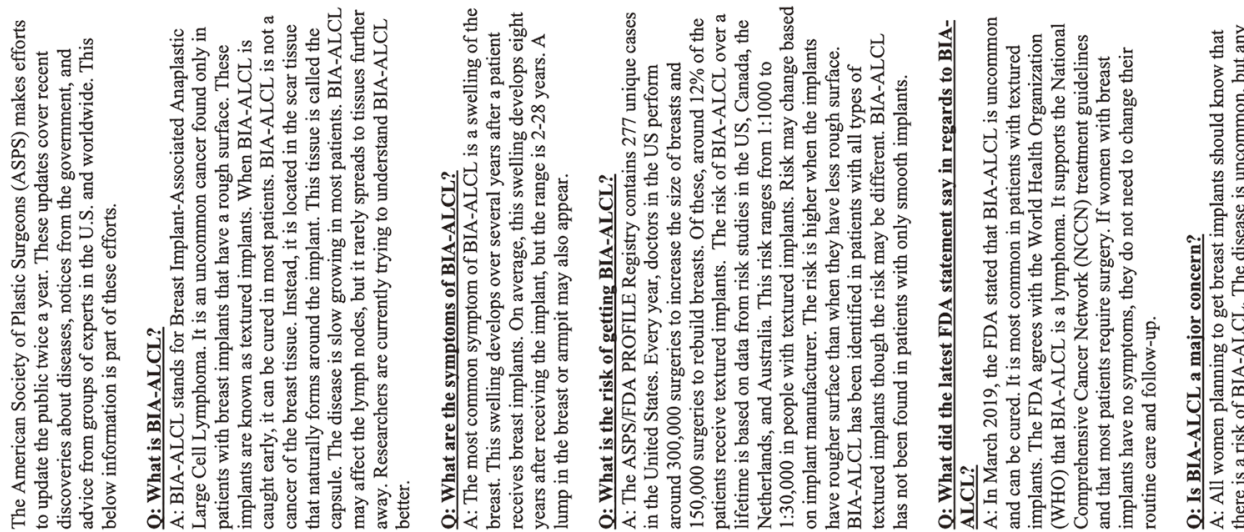

$\infty$
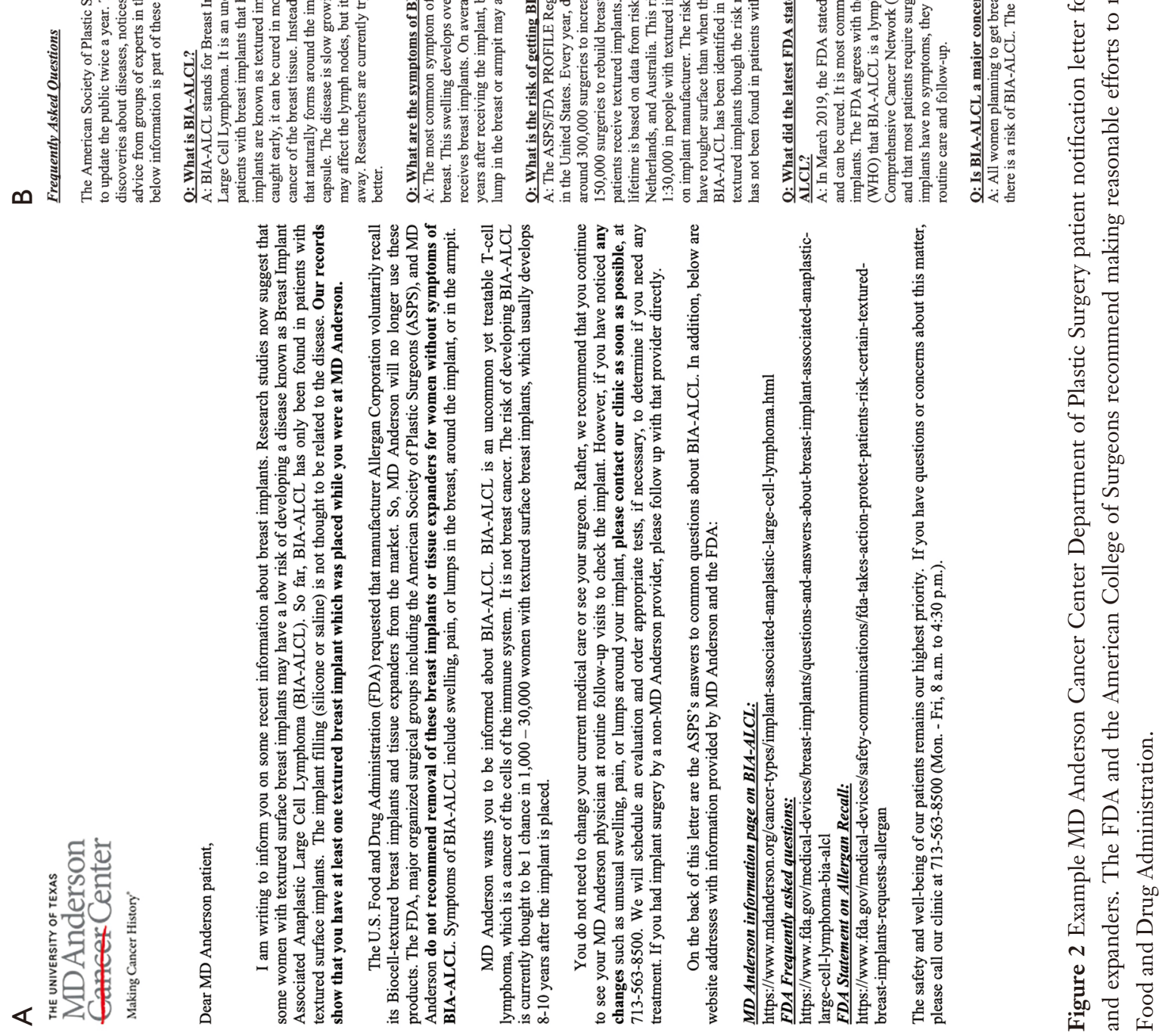
MD Anderson Cancer Center patients receiving a Biocell device and is demonstrated in Figure 2. Some academic institutions may not have the financial backing, clerical support, or access to electronic medical records to support this proactive approach. Private practice surgeons may also be met with significant roadblocks for similar reasons (55). Others have found notification of their patients an achievable goal, even in the private practice setting (39). Whether or not the approach to inform patients is retroactively taken with formal letters or notification, reasonable efforts should be employed to provide as much information to patients.

\section{Conclusions}

Physicians first and foremost should be advocates for their patients and be dedicated to intellectual honesty. In keeping with our commitment to patient safety, the strategies moving forward in regards to implant-based breast surgery should be marked by patient awareness and vigilance, and defined by evidence-based treatment. To fulfill our commitment to patient safety, patients should be provided education and access to reliable resources, so they can be informed when consenting for surgery, and more specifically should include notification for patients regarding the risk of BIA-ALCL. The FDA has taken steps to issue updated guidance recommendations that are forthcoming and will include a black box warning, mandated standard consent, recommendations for a patient decision checklist, improved patient labeling, and updated surveillance and screening guidelines. We should pursue evidence-based advocacy with transparent motives in order to clearly communicate and guide our patients.

\section{Acknowledgments}

Funding: None.

\section{Footnote}

Provenance and Peer Review: This article was commissioned by the Guest Editors (Charles E Butler, Carrie Chu, and Margaret Roubaud) for the series "New Frontiers in Breast Reconstruction" published in Gland Surgery. The article was sent for external peer review organized by the Guest Editors and the editorial office.

Conflicts of Interest: All authors have completed the ICMJE uniform disclosure form (available at http://dx.doi. org/10.21037/gs.2020.03.06). The authors have no conflicts of interest to declare.

Ethical Statement: The authors are accountable for all aspects of the work in ensuring that questions related to the accuracy or integrity of any part of the work are appropriately investigated and resolved.

Open Access Statement: This is an Open Access article distributed in accordance with the Creative Commons Attribution-NonCommercial-NoDerivs 4.0 International License (CC BY-NC-ND 4.0), which permits the noncommercial replication and distribution of the article with the strict proviso that no changes or edits are made and the original work is properly cited (including links to both the formal publication through the relevant DOI and the license). See: https://creativecommons.org/licenses/by-nc-nd/4.0/.

\section{References}

1. Coroneos CJ, Selber JC, Offodile AC, et al. US FDA Breast Implant Post-Approval Studies: Long Term Outcomes in 99,993 Patients. Ann Surg 2019;269:30-6.

2. Keech JA Jr, Creech BJ. Anaplastic T-cell lymphoma in proximity to a saline-filled breast implant. Plast Reconstr Surg 1997;100:554-5.

3. U.S. Food and Drug Administration, Archived Collection: Anaplastic Large Cell Lymphoma (ALCL) in women with breast implants: Preliminary FDA findings and analyses, January 2011. Available online: http://wayback. archive-it.org/7993/20171115053750/https:/www.fda. gov/MedicalDevices/ProductsandMedicalProcedures/ ImplantsandProsthetics/BreastImplants/ucm239996.htm. Accessed November 12, 2019.

4. Eaves F 3rd, Nahai F. Anaplastic large cell lymphoma and breast implants: FDA report. Aesthet Surg J 2011;31:467-8.

5. Swerdlow SH, Campo E, Pileri SA, et al. The 2016 revision of the World Health Organization classification of lymphoid neoplasms. Blood 2016;127:2375-90.

6. U.S. Food and Drug Administration. Medical device reports of breast implant-associated anaplastic large cell lymphoma. Available online: http://www.fda. gov/ MedicalDevices/ProductsandMedicalProcedures/ ImplantsandProsthetics/BreastImplants/ucm239996.htm. Accessed November 9, 2019.

7. Collins MS, Miranda RN, Medeiros LJ, et al. 
Characteristics and treatment of advanced breast implantassociated anaplastic large cell lymphoma. Plast Reconstr Surg 2019;143:41S-50S.

8. Ramos-Gallardo G, Cuenca-Pardo J, Rodríguez-Olivares $\mathrm{E}$, et al. Breast implant and anaplastic large cell lymphoma meta-analysis. J Invest Surg 2017;30:56-65.

9. Clemens MW, Miranda RN. Coming of age: Breast implant-associated anaplastic large cell lymphoma after 18 years of investigation. Clin Plast Surg 2015;42:605-13.

10. Clemens MW, Medeiros LJ, Butler CE, et al. Complete surgical excision is essential for the management of patients with breast implant-associated anaplastic largecell lymphoma. J Clin Oncol 2016;34:160-8.

11. Dashevsky BZ, Gallagher KM, Grabenstetter A, et al. Breast implant-associated anaplastic large cell lymphoma: Clinical and imaging findings at a large US cancer center. Breast J 2019;25:69-74.

12. Clemens MW, Horwitz SM. NCCN Consensus guidelines for the diagnosis and management of breast implant associated anaplastic large cell lymphoma. Aesthet Surg J 2017;37:285-9.

13. Clemens MW, Brody GS, Mahabir RC, et al. How to diagnose and treat breast implant-associated anaplastic large cell lymphoma. Plast Reconstr Surg 2018;141:586e-599e.

14. $\mathrm{Hu} \mathrm{H}$, Jacombs A, Vickery K, et al. Chronic biofilm infection in breast implants is associated with an increased T-cell lymphocytic infiltrate: Implications for breast implant-associated lymphoma. Plast Reconstr Surg 2015;135:319-29.

15. Jacombs A, Tahir $\mathrm{S}, \mathrm{Hu} \mathrm{H}$, et al. In vitro and in vivo investigation of the influence of implant surface on the formation of bacterial biofilm in mammary implants. Plast Reconstr Surg 2014;133:471e-480e.

16. Brody GS. The case against biofilm as the primary initiator of breast implant-associated anaplastic large cell lymphoma. Plast Reconstr Surg 2016;137:766e-767e.

17. Bizjak M, Selmi C, Praprotnik S, et al. Silicone implants and lymphoma: The role of inflammation. J Autoimmun 2015;65:64-73.

18. Miranda RN, Aladily TN, Prince HM, et al. Breast implantassociated anaplastic large-cell lymphoma: Long-term followup of 60 patients. J Clin Oncol 2014;32:114-20.

19. Clemens M. BIA-ALCL resources: By the numbers, and what they mean. Available online: https://www. plasticsurgery.org/for-medical-professionals/health-policy/ bia-alcl-physician-resources/by-the-numbers. Accessed November 10, 2019.
20. Bronz G. A comparison of naturally shaped and round implants. Aesthet Surg J 2002;22:238-46.

21. Friedman T, Davidovitch N, Scheflan M. Comparatie double-blind clinical study on round versus shaped cohesive gel implants. Aesthet Surg J 2006;26:530-6.

22. Macadam SA, Ho AL, Lennox PA, et al. Patient-reported satisfaction and health-related quality of life following breast reconstruction: a comparison of shaped cohesive gel and round cohesive gel implant recipients. Plast Reconstr Surg 2013;131:431-41.

23. Hidalgo DA, Weinstein AL. Intraoperative comparison of anatomical versus round implants in breast augmentation: a randomized controlled trial. Plast Reconstr Surg 2017;139:587-96.

24. Magnusson M, Beath K, Cooter R, et al. The epidemiology of breast implant-associated anaplastic large cell lymphoma in Australia and New Zealand confirms the highest risk for grade 4 surface breast implants. Plast Reconstr Surg 2019;143:1285-92.

25. Clemens MW. Discussion: The epidemiology of breast implant-associated anaplastic large cell lymphoma in Australia and New Zealand confirms the highest risk for grade 4 surface breast implants. Plast Reconstr Surg 2019;143:1295-7.

26. Loch-Wilkinson A, Beath KJ, Knight RJW, et al. Breast implant-associated anaplastic large cell lymphoma in Australia and New Zealand: high-surface-area textured implants are associated with increased risk. Plast Reconstr Surg 2017;140:645-54.

27. Adrada BE, Miranda RN, Rauch GM, et al. Breast implant-associated anaplastic large cell lymphoma: Sensitivity, specificity, and findings of imaging studies in 44 patients. Breast Cancer Res Treat 2014;147:1-14.

28. Montgomery-Goecker C, Fuda F, Krueger JE, et al. Immunophenotypic characteristics of breast implantassociated anaplastic large-cell lymphoma by flow cytometry. Cytometry B Clin Cytom 2015;88:291-3.

29. Lyapichev KA, Piña-Oviedo S, Medeiros LJ, et al. A proposal for pathologic processing of breast implant capsules in patients with suspected breast implant anaplastic large cell lymphoma. Mod Pathol 2020;33:367-79.

30. Tevis SE, Hunt KK, Clemens MW. Stepwise En Bloc Resection of Breast Implant-Associated Anaplastic Large Cell Lymphoma with Oncologic Considerations. Aesthetic Surgery Journal Open Forum 2019;1:ojz005.

31. National Comprehensive Cancer Network. About NCCN. Available online: www.nccn.org/about.

32. Johnson L, O’Donoghue JM, McLean N, et al. Breast 
implant associated large cell lymphoma: The UK experience. Recommendations on its management and implications for informed consent. Eur J Surg Oncol 2017;43:1393-401.

33. Rothman KJ. Epidemiology: An Introduction, 2nd Edition. Oxford University Press 2012:53.

34. Pittman TA, Fan KL, Rudolph MA. Anaplastic large cell lymphoma: Emerging consent and management patterns among American and international board-certified plastic surgeons. Plast Reconstr Surg 2016;138:811e-818e.

35. Doren EL, Miranda RN, Selber JC, et al. U.S. epidemiology of breast implant-associated anaplastic large cell lymphoma. Plast Reconstr Surg 2017;139:1042-50.

36. McCarthy CM, Loyo-Berrios N, Qureshi AA, et al. Patient registry and outcomes for breast implants and anaplastic large cell lymphoma etiology and epidemiology (PROFILE): Initial report of findings, 2012-2018. Plast Reconstr Surg 2019;143:65S-73S.

37. PSF PROFILE Registry Statistics. Available online: http:// www.thepsf.org/PROFILE. Accessed August November 11, 2019.

38. McGuire P, Reisman NR, Murphy DK. Risk factor analysis for capsular contracture, malposition, and late seroma in subjects receiving Natrelle 410 form-stable silicone breast implant. Plast Reconstr Surg 2017;139:1-9.

39. Clemens MW, McGuire P. Discussion: A prospective approach to inform and treat 1340 patients at risk for BIAALCL. Plast Reconstr Surg 2019;144:57-9.

40. Cordeiro PG, Ghione P, Nic A, et al. Risk of breast implant associated anaplastic large cell lymphoma (BIAALCL) in a cohort of 3546 women prospectively followed long term after reconstruction with textured breast implants. J Plast Reconstr Aesthet Surg 2020;73:841-6.

41. BIA-ALCL Resources: PROFILE and FDA data comparison. Oct 2019. Available online: https://www. plasticsurgery.org/documents/Health-Policy/ALCL/ PROFILE-Data-Summaries_Oct19.pdf. Accessed November 23, 2019.

42. U.S. Food and Drug Administration. The FDA requests Allergan voluntarily recall Natrelle Biocell textured breast implants and tissue expanders from the market to protect patients: FDA safety communication. Available online: https://www.fda.gov/medical-devices/safetycommunications/fda-requests-allergan-voluntarily-recallnatrelle-biocell-textured-breast-implants-and-tissue. Accessed November 10, 2019.

43. Teitelbaum S. AGN offers replacement implants. Available online: https://www.drteitelbaum.com/breast/breast- augmentation-revision/breast-implant-problems/alcl/pdf/ Customer_Letter_-_BIOCELL_Replacement_Warranty_ with_FAQ_update_7_30_19.pdf. Accessed November 10, 2019.

44. Allergan - Courthouse news. August 27, 2019. Available online: https://www.courthousenews.com/wp-content/ uploads/2019/08/Allergan.pdf. Accessed November 17, 2019.

45. AboutLawsuits.com - Allergan biocell class action lawsuit says manufacturer should replace breast implants, provide medical monitoring. August 22, 2019. Available online: https://aboutlawsuits-wpengine.netdna-ssl.com/wpcontent/uploads/2019-8-16-biocell-class-action.pdf. Accessed November 17, 2019.

46. U.S. Food and Drug Administration. Breast Implants. Available online: https://www.fda.gov/medical-devices/ implants-and-prosthetics/breast-implants. Accessed November 13, 2019.

47. U.S. Food and Drug Administration. Saline, silicone gel, and alternative breast implants: Guidance for industry and FDA staff. Available online: https://www.fda.gov/ regulatory-information/search-fda-guidance-documents/ saline-silicone-gel-and-alternative-breast-implants. Accessed November 14, 2019.

48. Carr LW, Roberts J, Potochny JD. How breast implant surface type is influenced by breast implant-associated anaplastic large cell lymphoma: A survey of the American Society of Plastic Surgeons. Ann Plast Surg 2019;82:S208-11.

49. Clemens MW, Miranda RN, Butler CE. Breast implant informed consent should include the risk of anaplastic large cell lymphoma. Plast Reconstr Surg 2016;137:1117-22.

50. del Carmen MG, Joffe S. Informed consent for medical treatment and research: A review. Oncologist 2005;10:636-41.

51. Horwitz SM, Ansell SM, Ai WZ, et al. T-cell lymphomas, Version 2.2018 featured updates to the NCCN Guidelines Feb 1 2018. J Natl Compr Canc Netw 2018;16:123-35.

52. Allergan Corp. Breast implant insert. Patient educational brochure on breast augmentation: Breast augmentation with NATRELLE silicone-filled breast implant. April 2009. Available online: https://www.fda.gov/media/80190/ download. Accessed November 17, 2019.

53. Allergan Corp. Breast implant insert. Patient educational brochure on breast augmentation: NATRELLE 410 highly cohesive anatomically shaped silicone-filled breast 
implants: Important factors breast augmentation patients should consider. Sept 2017. Available online: https:// media.allergan.com/actavis/actavis/media/allergan-pdfdocuments/labeling/natrelleus/410implants/natrelle-410aug-patient-ldoc-03824rev05.pdf. Accessed November 17, 2019.

Cite this article as: Parham CS, Hanson SE, Butler CE, Calobrace MB, Hollrah R, Macgregor T, Clemens MW. Advising patients about breast implant associated anaplastic large cell lymphoma. Gland Surg 2021;10(1):417-429. doi: 10.21037/ gs.2020.03.06
54. Roberts JM, Carr LW, Jones A, et al. A prospective approach to inform and treat 1340 patients at risk for BIAALCL. Plast Reconstr Surg 2019;144:46-54.

55. McCarthy CM. Discussion: A prospective approach to inform and treat 1340 patients at risk for BIA-ALCL. Plast Reconstr Surg 2019;144:60-1. 